\title{
Influências da depressão na psoríase: uma relação bidirecional
}

\author{
Influences of depression on psoriasis: a bidirectional relationship
}

Influencias de la depresión en la psoriasis: una relación bidireccional

\begin{abstract}
Mariana Olympio Rua ${ }^{1 *}$, Milena Ferreira Gandra ${ }^{2}$, Julia Oliveira Santos ${ }^{3}$, Rodrigo Lage Carneiro ${ }^{4}$, Juliana de Oliveira Silva ${ }^{5}$, Camila Silva Amaral ${ }^{6}$, Luana Caetano Barbosa ${ }^{2}$, Natália Bigossi Aguiar ${ }^{7}$, Giovanna Antonelli Melo Viol ${ }^{8}$, Débora Glenda dos Santos ${ }^{9}$.
\end{abstract}

\section{RESUMO}

Objetivo: O presente artigo buscou discutir a relação existente entre a psoríase e a depressão, pretendendo apresentar informações que correlacionam de que forma a piora da psoríase pode intensificar os sintomas depressivos, bem como o aumento dos sintomas depressivos pode levar à progressão da psicodermatose. Revisão bibliográfica: Foi demonstrado que o tratamento antidepressivo contribui para o manejo da patologia dermatológica, assim como inibidores de Fator de Necrose Tumoral (TNF), usados no tratamento da psoríase, colaboram com a melhora dos sintomas depressivos, uma vez que ambas têm relação com a liberação elevada de citocinas pró-inflamatórias. Entretanto, o mecanismo exato de como a inflamação intervém nas duas patologias ainda não está elucidado por completo. Considerações finais: Assim, é necessário um tratamento multidimensional e que leve em consideração o aspecto psicológico, social e de bem-estar físico, visto que a psoríase não apresenta cura e o tratamento da depressão minimizaria os efeitos adversos dessa dermatose.

Palavras-chave: Psoríase, Depressão, Inflamação.

\begin{abstract}
Objective: This article sought to discuss the relationship between psoriasis and depression, aiming to present information that correlates how the worsening of psoriasis can intensify depressive symptoms, as well as the increase in depressive symptoms can lead to the progression of psychodermatosis. Bibliographic review: It has been shown that antidepressant treatment contributes to the management of dermatological pathology, as well as Tumor Necrosis Factor (TNF) inhibitors, used in the treatment of psoriasis, collaborate with the improvement of depressive symptoms, since both are related to the elevated release of pro-inflammatory cytokines. However, the exact mechanism of how inflammation intervenes in both pathologies is not yet fully elucidated. Final considerations: Therefore, multidimensional treatment is necessary, taking into account the psychological, social and physical well-being aspects, since psoriasis has no cure and the treatment of depression would minimize the adverse effects of this dermatosis.
\end{abstract}

Key words: Psoriasis, Depression, Inflammation.

\section{RESUMEN}

Objetivo: Este artículo buscó discutir la relación entre la psoriasis y la depresión, con el objetivo de presentar información que correlacione cómo el empeoramiento de la psoriasis puede intensificar los síntomas depresivos, así como el aumento de los síntomas depresivos puede conducir a la progresión de la psicodermatosis. Revisión bibliográfica: Se ha demostrado que el tratamiento antidepresivo contribuye al

\footnotetext{
${ }^{1}$ Universidade Vila Velha (UVV), Vila Velha - ES. *E-mail: marilympio@gmail.com

2 Centro Universitário de Caratinga (UNEC), Caratinga - MG.

3 Universidade de Uberaba (UNIUBE), Uberaba - MG.

${ }^{4}$ Pontifícia Universidade Católica de Minas Gerais (PUC Minas), Poços de Caldas - MG.

${ }^{5}$ Universidade Federal do Pará (UFPA), Belém - PA.

${ }_{6}$ Universidad de Ciencias Empresariales y Sociales (UCES), Buenos Aires - AR.

${ }^{7}$ Centro Universitário do Espírito Santo (UNESC), Colatina - ES.

${ }^{8}$ Centro Universitário de Volta Redonda (UNIFOA), Volta Redonda - RJ.

9 Universidade Potiguar (UNP), Natal - RN.
} 
manejo de la patología dermatológica, así como los inhibidores del Factor de Necrosis Tumoral (TNF), utilizados en el tratamiento de la psoriasis, colaboran con la mejoría de los síntomas depresivos, ya que ambos están relacionados con la alta liberación de citocinas proinflamatorias. Sin embargo, el mecanismo exacto de cómo interviene la inflamación en ambas patologías aún no está completamente aclarado. Consideraciones finales: Por tanto, es necesario un tratamiento multidimensional, teniendo en cuenta los aspectos psicológicos, sociales y de bienestar físico, ya que la psoriasis no tiene cura y el tratamiento de la depresión minimizaría los efectos adversos de esta dermatosis.

Palabras clave: Psoriasis, Depresión, Inflamación.

\section{INTRODUÇÃO}

A psoríase é uma doença crônica inflamatória da pele, que causa lesões pruriginosas, eritematosas, pápulas descamativas e placas, na qual as células da epiderme se juntam, formando manchas e escamas secas causando prurido, resultantes de interações genéticas como, fatores ambientais e o sistema imunitário, estando fortemente ligada a depressão e distúrbios emocionais (NESTLE N, et al., 2009).

A depressão e outros fatores psicológicos foram evidenciados como partes determinantes para o desencadeamento do início e da gravidade da psoríase. Além disso, as feridas da doença, que podem causar consequências nas relações sociais do paciente, e consigo mesmo, diminuindo sua autoestima e causandoIhe constrangimento (REICH A, et al., 2016).

Essa patologia dermatológica, a qual gera mudanças e lesões no epitélio, pode provocar estigmas, devido às alterações de aparência que causam. Com isso, muitos pacientes desenvolvem problemas sociais que estão muito ligados ao desencadeamento e intensificação dos sintomas depressivos. Da mesma forma em que a psoríase pode desencadear alterações psicológicas, a depressão, por poder causar alterações no sistema imune, influencia diretamente no quadro dessa patologia dermatológica (JESUS NA, et al., 2016).

A depressão é um transtorno grave e prevalente, com incidência de 4,4\% a $20 \%$ na população total, sendo uma doença que causa prejuízos à qualidade de vida do paciente que não obteve o tratamento adequado. $O$ distúrbio em questão é três vezes mais comum em pessoas com doenças crônicas, como a psoríase, do que na população em geral. Nesse sentido, nota-se que ambas as doenças estão associadas a uma série de eventos adversos, tais como aumento da mortalidade, menor qualidade de saúde e maiores custos em serviços de saúde; podendo, inclusive, interferir no desenvolvimento uma da outra (VALCARCEL M, et al., 2016).

O presente artigo tem como objetivo avaliar de que forma a depressão contribui para o desenvolvimento da psoríase. Ademais, pretende reconhecer a influência que o tratamento da depressão exerce na psoríase e vice-versa.

\section{REVISÃO BIBLIOGRÁFICA}

\section{Psoríase}

A psoríase é uma enfermidade inflamatória crônica sistêmica que acomete primordialmente a pele, as mucosas e as unhas, podendo também comprometer as articulações. Acredita-se que a psoríase é uma doença inflamatória imunomediada com um componente psiquiátrico em sua patogênese (BRUFAU R, et al., 2010).

A manifestação da psoríase ocorre principalmente entre os 15 aos 20 anos de idade, ou em um segundo pico de ocorrência entre 55 e 60 anos de idade. O desenvolvimento da doença antes dos 40 anos está associado, muitas vezes, ao fator genético, além de apresentar um grande envolvimento psicológico (MOREIRA FFB, et al., 2016).

A psoríase está associada a vários distúrbios afetivos, em particular à ansiedade e à depressão. De acordo com Dowlatshahi EA, et al. (2014), a prevalência de depressão como comorbidade da psoríase é estimada 
entre $20 \%$ e $30 \%$, atingindo até $62 \%$ em alguns estudos. Kurd SK, et al. (2010) confirmaram esses achados em uma grande série de casos de pacientes com psoríase: foi encontrada uma alta prevalência de depressão, ansiedade e ideação suicida (39\%, 31\% e 44\%, respectivamente), que aumentaram nos casos mais graves da doença. Isso pode ser comprovado por pesquisas que relatam que cerca de 40 a $90 \%$ dos pacientes portadores de psoríase possuem algum distúrbio psicológico (MOREIRA FFB, et al., 2016).

O estresse psicológico se apresenta como um dos fatores mais frequentemente responsáveis pelo desencadeamento ou piora da doença, geralmente, quando acompanhado de situações de preocupação, ansiedade, irritabilidade ou nervosismo. Isso pode ocorrer porque a dermatose acaba configurando uma forma do corpo expressar o que não está conseguindo suportar, e exterioriza por meio da somatização, em que a dor psíquica começa a se manifestar no corpo através de lesões físicas na pele, assim como ocorre na psoríase (MELO MSB, et al., 2019).

Observou-se que a hiperproliferação descontrolada de queratinócitos na psoríase é acompanhada de abrangente atividade inflamatória. Esta se inicia com uma forte estimulação dos linfócitos $T$, por meio da apresentação de antígenos próprios pelas células dendríticas, inicialmente para os linfócitos $T$ auxiliares (TCD4+) na derme e, posteriormente, para os linfócitos T citotóxicos (TCD8+) na epiderme. A partir disso, citocinas são liberadas gerando o desencadeamento do processo inflamatório: (1) Interleucinas (IL-1, IL-6, IL8, IL-17); (2) Fator de Necrose Tumoral a (TNF-a) e (3) Interferon (IFN). Com o avanço deste processo próinflamatório, os queratinócitos tornam-se ainda mais reativos, e, por sua vez, também aumentam sua produção autócrina de citocinas e fatores de crescimento nos locais de lesões. Estas proteínas liberadas aumentam a ação e o recrutamento de células $T$, ampliam o influxo de neutrófilos, além de permitirem alterações vasculares e a proliferação ainda maior de queratinócitos (JESUS NA, et al., 2016).

Em relação aos aspectos clínicos, a psoríase caracteriza-se por possuir evolução crônica com tendência a acentuações ou recidivas, decorrente da hiperproliferação de queratinócitos. A lesão tem predileção pelo couro cabeludo, unhas, cotovelos, joelhos, e tronco, sendo representada por uma placa eritemato-escamosa saliente em relação à superfície da pele. As escamas estão superpostas, apresentam cor eritematosa e podem ser destacadas com facilidade, mediante a raspagem da lesão. Ademais, em relação à morfologia, as lesões possuem diversos tamanhos, desde gotas, até padrões policíclicos (MELO MSB, et al., 2019).

O diagnóstico da psoríase se baseia no histórico e nos sintomas clínicos do paciente. Contudo, pode-se utilizar a biopsia para a confirmação do diagnóstico, além da prevenção e tratamento a fim de retardar sua recidiva (MESQUITA PE, 2013).

\section{Depressão}

Existem afetações dermatológicas que possuem uma base fisiopatológica dérmica primária, porém outras estão influenciadas por fatores psicossociais e transtornos psiquiátricos, as chamadas psicodermatoses (ACOSTA J, et al., 2012). A depressão, um dos transtornos que pode afetar as psicodermatoses, é um tipo de transtorno afetivo que provoca alterações mentais, corporais e distúrbios de humor. É um conjunto de sintomas que pode durar semanas, meses e perdurar por anos, interferindo de forma significativa na vida pessoal, social e profissional do indivíduo (JARDIM S, 2011).

Segundo a $5^{\mathfrak{a}}$ edição do Manual Diagnostico e Estatístico de Transtornos Mentais (DSM-V), pode-se dizer que um paciente tem depressão quando experienciou pelo menos cinco dos seguintes sintomas, durante período igual ou superior a 2 semanas: perda de interesse pelas atividades, humor depressivo, distúrbios do sono, do apetite/peso, alterações psicomotoras, sensação de culpa, perda de energia, dificuldade de concentração, incapacidade de tomar decisões, pensamentos suicidas ou relacionados com a morte (AMERICAN PSYCHIATRIC ASSOCIATION (APA), 2013). De acordo com a Classificação de Transtornos Mentais e de Comportamento da CID-10, a depressão é um transtorno mental frequente, além de ser a principal causa de incapacidade em todo o mundo. Estima-se que mais de 300 milhões de pessoas, de todas as idades, sofram com esse transtorno (ORGANIZAÇÃO MUNDIAL DA SAÚDE (OMS), 1993).

Embora existam muitas teorias para explicar todos os contextos da doença, nenhuma delas é conclusiva e totalmente abrangente. As teorias envolvem fatores genéticos, fatores biológicos, como a redução de 
neurotransmissores como a noradrenalina, serotonina, dopamina e acetilcolina, e também os hormônios que regulam o estresse (VAZQUEZ MB, et al., 2019).

A relação dos transtornos mentais e as doenças dermatológicas é bidirecional, sendo observado que grande parte da literatura relacionada acerca da depressão aponta para sua associação frequente em pacientes com psoríase. Em uma extensa revisão sistemática, Dowlatshahi EA, et al. (2014) constataram que $19 \%$ dos pacientes estudados com psoríase preenchiam os critérios da 4⿳a edição do Manual Diagnóstico e Estatístico de Transtornos Mentais (DSM-IV) para depressão.

\section{Relação entre depressão e psoríase}

É difícil determinar até que ponto os sintomas de depressão em pacientes com distúrbios dermatológicos são devidos à doença inflamatória cutânea subjacente em comparação ao impacto psicossocial de viver com uma doença crônica. A natureza desfigurante e visível das doenças inflamatórias crônicas da pele contribui para o impacto psicossocial. Porém, a prevalência de depressão na psoríase é maior do que em doenças cutâneas desfigurantes, como a ictiose (FARZANFAR D, et al., 2018). Existe uma relação bidirecional entre a depressão e a psoríase, ou seja, ambas estão interligadas por meio de mecanismos inflamatórios. $\mathrm{Na}$ depressão foi encontrado um aumento da concentração de citocinas pró-inflamatórias sistêmicas, as quais migram para a pele e causam a psoríase em indivíduos suscetíveis (LESCANO PKP, 2018).

Citocinas pró-inflamatórias associadas a diversas doenças de pele podem estar causalmente associadas à sintomatologia depressiva coexistente. Níveis elevados de citocinas circulantes podem ter um impacto psicológico. Sendo assim, pode-se dizer que há uma potencial relação entre mediadores inflamatórios derivados da pele que afetam negativamente o bem-estar psicológico e a depressão que leva ao aumento da inflamação da pele, criando um ciclo de exacerbação crônica (FARZANFAR D, et al., 2018).

Foi descoberto que indivíduos com depressão possuem níveis mais elevados de citocinas próinflamatórias, especialmente TNF- $\alpha$ e a IL-6, sugerindo que essas citocinas provavelmente estão envolvidas no desenvolvimento e manutenção da depressão, mesmo em pacientes sem doenças inflamatórias crônicas subjacentes. Essas citocinas, após ultrapassarem a barreira hematoencefálica, associam-se com 0 metabolismo alterado da serotonina, norepinefrina e dopamina em áreas do cérebro que regulam as emoções e as funções psicomotoras. Além disso, essas citocinas também estimulam o eixo hipotálamo-hipófiseadrenal, aumentando os níveis do hormônio cortisol, frequentemente elevado em pacientes com depressão (KANNAN S, et al., 2013).

Embora a barreira hematoencefálica sirva para impedir a entrada dessas citocinas, três mecanismos foram propostos para a entrada desses mediadores inflamatórios no sistema nervoso central, sendo: uma barreira hematoencefálica com vazamento em indivíduos com depressão; ativação das células endoteliais revestidoras da vasculatura cerebral, causando produção de citocinas dentro da barreira; e mediadores inflamatórios ligando-se a receptores associados ao nervo vago, que podem sinalizar alterações inflamatórias no cérebro (PATEL N, et al., 2017).

Ainda que a psoríase e a depressão possuam em suas respectivas patogêneses o seu próprio conjunto único de citocinas, a IL-17 e o TNF- $\alpha$ estão presentes em ambas as doenças, comprovando a existência de uma ligação fisiopatológica entre elas (PATEL N, et al., 2017).

As citocinas interrompem a síntese de serotonina por meio de alguns mecanismos. O interferon alfa (IFNa) serve como um potente indutor de IL-6, IL-1 $\beta$ e TNF- $\alpha$, que geram alterações no metabolismo da serotonina. Este IFN- $\alpha$, juntamente com a administração de IL-2, aumenta a atividade da indoleamina 2,3 dioxigenase, uma enzima que degrada triptofano em quinurenina. Decorrente deste processo, a serotonina, por ser derivada do triptofano, sofre uma diminuição de sua produção. Ademais, os catabólitos do triptofano podem alterar a função do hormônio liberador de corticotropina e induzir sintomas depressivos. Já a IL-6 e o TNF- $\alpha$ facilitam a quebra da serotonina, diminuindo seus níveis no cérebro (PATEL N, et al., 2017).

Por conseguinte, pode-se dizer que a inflamação tem um papel fundamental e notório em ambas as patologias. No entanto, o mecanismo exato de como ocorre a intervenção na depressão e na psoríase ainda não está elucidado por completo. Os dermatologistas devem estar conscientes do potencial de melhora na 
qualidade de vida do paciente ao abordar a dimensão psíquica das enfermidades da pele (VALCARCEL M, et al., 2016; LESCANO, 2018).

\section{Tratamento multidisciplinar da psoríase}

No que se refere ao tratamento da psoríase, é importante apontar que essa dermatose não tem cura, ou seja, as medidas terapêuticas visam apenas amenizar as lesões já existentes. (MOREIRA FFB, et al., 2016). Existem várias modalidades de tratamento, entre agentes terapêuticos tópicos e sistêmicos. A escolha da terapia depende da extensão e da severidade da doença, da segurança dos agentes terapêuticos, acessibilidade ao tratamento, qualidade de vida e vontade do paciente quanto ao tipo de terapia, embora nenhum medicamento usado seja totalmente seguro e eficaz (RODRIGUES A e TEIXEIRA R, 2009; PRADHAN M, et al., 2013).

A somatização é uma forma do indivíduo expressar de forma física sua incapacidade de conter a insatisfação ou o desconforto perante os desafios do cotidiano, levando ao desencadeamento de diversas doenças dermatológicas decorrentes da sintomatologia de suas próprias emoções (MOREIRA FFB, et al., 2016). Diante disso, percebeu-se que os fatores psiquiátricos e psicológicos são importantes no momento da avaliação e manejo das psicodermatoses, tais como acne, alopecia areata, rosácea, urticária, dermatite atópica, vitiligo vulgar e psoríase (HASSAN I, et al., 2019).

Nesse sentido, é necessária uma intervenção psicológica com o objetivo de amenizar sintomas e a qualidade de vida do paciente, associado a um tratamento farmacêutico das lesões (MOREIRA FFB, et al., 2016). É importante ressaltar a necessidade de fornecer apoio psicossocial para pacientes com psoríase, visto que a abordagem eficaz reside em um acompanhamento multidimensional que proporciona ao paciente bem-estar físico, social e psicológico (JENSEN P, et al., 2016). Tendo em consideração o efeito da depressão na psoríase, observou-se que a terapia antidepressiva contribui no tratamento dessa doença, evidenciando, com isso, uma íntima relação entre o manejo da psoríase e da depressão (KOURIS A, et al., 2017).

Estudos randomizados, com algumas limitações a serem consideradas, nos quais relacionaram medicações, primariamente utilizadas no tratamento da psoríase, que parecem influenciar de maneira positiva em ambas as doenças. Nesses, o Adalimumabe apresentou melhora no aspecto físico, social e psicológico. No tratamento de pacientes com psoríase moderada a grave, o Ustecinumab demonstrou uma considerável redução dos sintomas depressivos. Ademais, resultados confirmaram que independente da gravidade da psoríase, os inibidores de TNF diminuem a gravidade dos sintomas depressivos, além de apresentarem efeitos positivos na função cognitiva e no humor. Em outros estudos, o Infliximabe e o Etanercepte apresentaram resultados semelhantes as medicações citadas (GONZÁLEZ-PARRA S E DAUDEŃ E, 2019).

Um estudo observacional foi realizado na Árabia Saudita, com a finalidade de observar a atuação do medicamento Adalimumabe em sintomas depressivos associados à psoríase moderada a grave. Dos 45 pacientes que receberam tratamento com o medicamento, $97,8 \%$ apresentou melhora clinicamente significativa dos sintomas de ambas as comorbidades (ELGENDI A, et al., 2015). Observou-se, também, que as concentrações anormais de substâncias químicas, incluindo a melatonina, foram encontradas em níveis baixos em pacientes com depressão e psoríase. Entretanto, a regulação química com melatonina, teve efeitos positivos nesses pacientes, reduzindo a morbidade das doenças (DANIYAL A, et al., 2018).

O impacto psicossocial da psoríase é tão importante quanto suas consequências físicas, contribuindo, dessa forma, para a morbidade geral da doença (KOURIS A, et al.,2017). Portanto, vale ressaltar a relevância do tratamento multidisciplinar, principalmente quando coexistem ambas as doenças. O manejo eficaz deve ser multidimensional e multifatorial, considerando os aspectos físicos, psicológicos e sociais do paciente. $\mathrm{O}$ estudo dessas características individuais pode auxiliar a profilaxia, o tratamento e a forma de manejar os fatores desencadeadores e/ou potencializadores dessas doenças (SILVA KS E SILVA EAT, 2007). O tratamento não-medicamentoso é fundamental tanto para a manutenção da qualidade de vida, quanto para a prevenção de episódios de agudização das doenças. Hábitos de vida saudáveis, como: dieta adequada, prática regular de exercícios físicos, perda de peso e diminuição da ingesta alcoólica contribuem para 0 tratamento e prevenção de ambas (GONZÁLEZ-PARRA S E DAUDÉN E, 2019). 


\section{CONSIDERAÇÕES FINAIS}

A partir dos dados supracitados, considerou-se que a depressão e a psoríase têm relação bidirecional com ligação fisiopatológica. Foi demonstrado que o tratamento antidepressivo contribui para o manejo da patologia dermatológica, assim como, inibidores de TNF, usadas no tratamento da psoríase, colaboram com o da depressão, uma vez que ambas têm relação com a liberação elevada de citocinas pró-inflamatórias. Todavia, o mecanismo exato de como a inflamação atua nas duas patologias ainda não está completamente esclarecido. O tratamento medicamentoso da psoríase não apresenta objetivo de cura, mas de melhora da qualidade de vida do paciente ou manutenção da remissão. Portanto, foi evidenciado que o tratamento multidisciplinar trouxe vantagens na terapêutica da psicodermatose, atentando-se aos aspectos físicos, psicológicos e sociais do paciente.

\section{REFERÊNCIAS}

1. ACOSTA J, et al. Depression and depressive symptoms in outpatients of a Dermatology's service in a general hospital of Lima. Rev Neuropsiquiatr, 2010; 73(4): 126-135.

2. AMERICAN PSYCHIATRIC ASSOCIATION (APA). Diagnostic and Statistical Manual of Mental Disorders, Fifth Edition (DSM-V). Arlington, VA: American Psychiatric Association, 2013; 948p.

3. BRUFAU R, et al. Estilos de personalidad en pacientes con psoriasis. Anales de Psicologia, 2010; 26(2): 335-44.

4. DANIYAL A, et al. Pro-inflammatory Cytokines, Biomarkers, Genetics and the Immune System: A Mechanistic Approach of Depression and Psoriasis. Rev Colombiana de Psiquiatria, 2018; 47(3): 177-186.

5. DOWLATSHAHI EA, et al. The prevalence and odds of depressive symptoms and clinical depression in psoriasis patients: a systematic review and meta-analysis. J Invest Dermatol; 2014; 134(6): 1542-1551.

6. DUARTE GV, et al. Psoriasis and obesity: literature review and recommendations for management. An Bras Dermatol, 2010; 85: 355-60.

7. ELGENDI A, et al. Depression Over Psoriasis: Assessment of associated Relief by addition of ADalimumab for the treatment of Psoriasis: Observational study. Journal of Dermatology \& Dermatologic Surgery 2015; 19: 15-20.

8. FARZANFAR D, et al. Inflammation: A Contributor to Depressive Comorbidity in Inflammatory Skin Disease. Skin Pharmacol Physiol, 2018; 31: 246-251.

9. GONZÁLEZ-PARRA S, DAUDÉN E. Psoriasis na Depresion: The role of inflammation. Revista ACTAS DermoSifiliográficas, 2019; 110(1): 12-19.

10. HASSAN I, et al. Psychodermatoses in children. Indian J Paediatr Dermatol 2019; 20: 306-14.

11. JARDIM S. Depressão e trabalho: Ruptura de Laço Social. Revista Brasileira de Saúde Ocupacional, 2011 ; 36 : 8492.

12. JENSEN P, et al. Psoriasis and New-onset depression: a Danish nationwide cohort study. Acta Derm Venereol, 2016; 96: 39-42.

13. JESUS NA, et al. Impacto da psoríase na qualidade de vida dos pacientes em tratamento: uma revisão sistemática da literatura. Revista InterScientia. 2016; 4(1): 37-41.

14. KANNAN S, et al. The role of tumor necrosis factor-alpha and other cytokines in depression: what dermatologists should know. Journal of Dermatological Treatment, 2013; 24: 148-152.

15. KOURIS A, et al. Psychological parameters of psoriasis, Rev Psychiatriki, 2017, 28: 54-59.

16. KURD SK, et al. The risk of depression, anxiety, and suicidality in patients with psoriasis: A population-based cohort study. Arch Dermatol, 2010; 146: 891-5.

17. LESCANO PKP, KAREN P. Bidirectional relationship of the skin pathologies with mental disorders. Rev Cient Cienc Med, 2018; 21(1): 84-89.

18. MELO MSB, et al. Influência de fatores emocionais nas doenças crônicas de pele: o estresse como gatilho para o desenvolvimento, reincidência ou agravamento da psoríase. Revista Multidisciplinar e de Psicologia, 2019; 13(46): 584-608.

19. MESQUITA PE. Psoríase: Fisiopatologia e Terapêutica. Dissertação (Mestrado em Ciências Farmacêuticas) Faculdade de Ciências da Saúde. Universidade Fernando Pessoa, Portugal, 2013; 54 p.

20. MOREIRA FFB, et al. A influência do estresse no desencadeamento e evolução da psoríase, Brazilian Journal of Surgery and Clinical Research, 2016, 15(1): 87-90.

21. NESTLE FO, Depression Is Associated with an Increased Risk of Psoriatic Arthritis among Patients with Psoriasis: A Population-Based Study, Journal of Investigative Dermatology, 2017; 137: 828-835;

22. ORGANIZAÇÃO MUNDIAL DA SAÚDE (OMS). Classificação de Transtornos Mentais e de Comportamento da CID10. Porto Alegre: Artmed, 1993; 105p.

23. PATEL N, et al. Psoriasis, Depression, and Inflammatory Overlap: A Review. Am J Clin Dermatol, 2017; $18: 613-620$.

24. PRADHAN M, et al. Novel colloidal carriers for psoriasis: current issues, mechanistic insight and novel delivery approaches. Journal of Controlled Release, 2013; 170: 380-395.

25. REICH A, Interplay of Itch and Psyche in Psoriasis: An Update, ActaDV, 2016; 217: 55-57.

26. RODRIGUES A, TEIXEIRA R. Desvendando a psoríase. RBAC, 2009; 41:(4), 303-309.

27. VALCARCEL M, et al. Psoriasis y depresíon. Rev GPU, 2016; 12(2): 168-173.

28. VAZQUEZ MB, et al. Prevalencia de los síntomas de depresión en pacientes con psoriasis en la Argentina. Dermatología Argentina, 2019; 25(2): 72-77. 